\title{
Recurrent blistering of the fingers as a sign of carpal tunnel syndrome: a first report from Japan
}

\section{Mai Endo, Toshiyuki Yamamoto}

\author{
Department of Dermatology, Fukushima Medical University Hikarigaoka 1, Fukushima 960-1295, Japan
}

Corresponding author: Dr. Mai Endo, E-mail: enmai04@fmu.ac.jp

Sir,

Carpal tunnel syndrome is an entrapment neuropathy caused by compression of the median nerve. Dermatological signs associated with carpal tunnel syndrome are rare, and to date, ulceration, blistering, hypohidrosis, Raynaud's phenomenon, and irritant contact dermatitis have been reported. Among them, blistering of the fingers is extremely rare. We herein report a patient with carpal tunnel syndrome, who developed recurrent blisters on the fingers.

A 75-year-old woman, who was suffering from carpal tunnel syndrome for 15 years, was referred to our department complaining of skin eruptions on the fingers. For the previous 6 years, blisters repeatedly appeared on the digits of her right hand and healed in about a month after ulceration. Therefore, she was referred to our department. On physical examination, she had no blisters other than an erosion in her right third finger (Fig. 1). Symptoms of local infection were not observed. The site where the blistering occurred had become reddish after erosion. There were no blisters other than those on the fingers. She denied Raynaud's phenomenon, and neither finger coldness nor swelling was observed. Just before the blisters appeared, she had a complaint of slight pain at the same site. She stated that blisters had occurred on all fingers except the little finger. After a few months, the blister occurs and collapses in a few days. It is not related to the season, there is no special timing, and there was no history of trauma or local infection.

Cutaneous manifestation associated with carpal tunnel syndrome has been poorly described. To date, there are several cases of carpal tunnel syndrome showing cutaneous manifestations in English literature [1-6]; however, to our knowledge, there are no reports from Japan. In all cases, the lesions were found in fingers, and in severe cases, bone and nail changes were also induced [3]. In cases that underwent surgical decompression for carpal tunnel syndrome, skin lesions had disappeared $[1,2,4]$. In the present case, blisters repeatedly appeared on the right third finger innervated by the median nerve, although other fingers were also involved. According to the patient's complaint, the blisters waxed and waned; however, the triggering event of the blister appearance was unknown. Unfortunately, we could not have any chance to perform biopsy; however, bullous pemphigoid was excluded because bullous formation was restricted to the fingers and disease course showed recurrent spontaneous regression and relapse. Other diseases such as diabetic bulla, blistering digital dactylitis, and herpetic whitlow were all differentiated.

Although the mechanism by which blisters can occur is not disclosed, ischemic changes due to autonomic neuropathy and vasomotor dysfunction are suggested to play a major role, and minor trauma and secondary infection may also be involved. On the other hand, nerve compression in carpal tunnel syndrome is a privileged site to become acquired immunocompromised district [7]. Baroni et al. speculated that the areas innervated by median nerve fiber compression are an obstacle to the normal passage of immunocompetent cells through lymphatic channels or an interference with neuropeptide signaling by peripheral nerves to the membrane receptors of immunocompetent cells, caused by nerve 


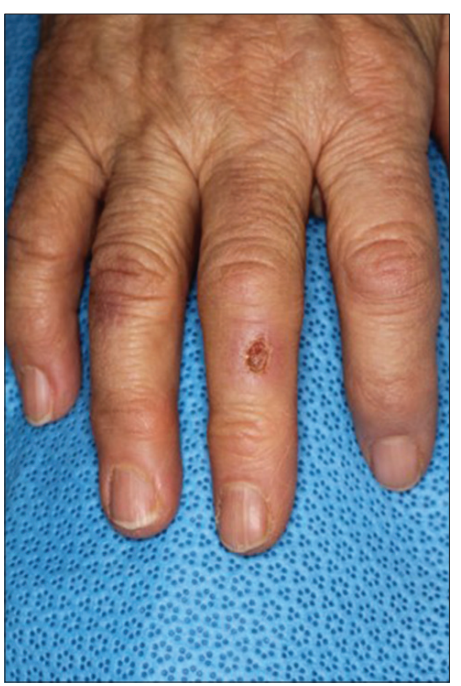

Figure 1: Erosive lesion on the dorsa of the third finger of the right hand.

fiber compression, and dysregulated neuropeptide release may induce various manifestations including blister formation [5].

In conclusion, we reported a case of recurrent blistering of the fingers as a sign of carpal tunnel syndrome. Digital ulcers can be the first manifestation of carpal tunnel syndrome [6]. When we see recurrent blisters of the fingers, carpal tunnel syndrome should be considered as one of the differential diagnoses.

\section{Consent}

The examination of the patient was conducted according to the Declaration of Helsinki principles.

\section{REFERENCES}

1. Aratari E, Regesta G, Rebora A. Carpal tunnel syndrome appearing with prominent skin syndromes. Arch Dermatol. 1984;120:517-9.

2. Cox NH, Large DM, Paterson WD, Ive FA. Blisters, ulceration and autonomic neuropathy in carpal tunnel syndrome. Br J Dermatol. 1992;126:611-3.

3. Tosti A, Morelli R, D'Alessandro R, Bassi F. Carpal tunnel syndrome presenting with ischemic skin lesions, acroosteolysis, and nail changes. J Am Acad Dermatol. 1993;29:287-90.

4. Foti C, Romita P, Vestita M. Unusual presentation of carpal tunnel syndrome with cutaneous signs: a case report and review of the literature. Immunopharmacol Immunotoxicol. 2011;33:751-3.

5. Baroni A, Piccolo V, Russo T, Ruocco V. Recurrent blistering of the fingertips as a sign of carpal tunnel syndrome: An effect of nerve compression. Arch Dermatol. 2012;148:545-6.

6. Perdan-Pirkmajer K, Praprotnik S, Tomšič M. Digital ulcers as the first manifestation of carpal tunnel syndrome. Rheumatol Int. 2011;31:685-6.

7. Ruocco V, Ruocco E, Piccolo V, Brunetti G, Guerrera LP, Wolf RW. The immunocompromised district in dermatology: a unifying pathogenic view of the regional immune dysregulation. Clin Dermatol. 2014;32:569-76.

Copyright by Mai Endo, et al. This is an open-access article distributed under the terms of the Creative Commons Attribution License, which permits unrestricted use, distribution, and reproduction in any medium, provided the original author and source are credited.

Source of Support: Nil, Conflict of Interest: None declared. 